\title{
Cluster scaling relations from cosmological hydrodynamic simulations in a dark-energy dominated universe
}

\author{
N. Aghanim ${ }^{1}$, A. C. da Silva ${ }^{1,2}$, and N. J. Nunes ${ }^{3}$ \\ 1 IAS, CNRS \& Université Paris Sud, Bâtiment 121, 91405 Orsay, France \\ e-mail: nabila.aghanim@ias.u-psud.fr \\ 2 Centro de Astrofísica, Universidade do Porto, Rua das Estrelas, 4150-762 Porto, Portugal \\ e-mail: asilva@astro.up.pt \\ 3 Department of Applied Mathematics and Theoretical Physics, Wilberforce Road, Cambridge, CB3 0WA, UK \\ e-mail: nunes@damtp.cam.ac.uk
}

Received 28 July 2008 / Accepted 20 November 2008

\begin{abstract}
Context. Clusters are potentially powerful tools for cosmology provided their observed properties, such as the Sunyaev-Zel'dovich (SZ) or X-ray signals, can be translated into physical quantities like mass and temperature. Scaling relations are the appropriate means to perform this translation. It is, therefore, important to understand their evolution and their modifications with respect to the physics and to the underlying cosmology.

Aims. In this spirit, we investigate the effect of dark energy on the X-ray and SZ scaling relations. The study is based on the first hydrosimulations of cluster formation for diferent models of dark energy. We present results for four dark-energy models which differ from each other by their equations-of-state parameter, $w$. Namely, we use a cosmological constant model $w=-1$ (as a reference), a perfect fluid with constant equation of state parameter $w=-0.8$ and one with $w=-1.2$ and a scalar field model (or quintessence) with varying $w$.

Methods. We generate $N$-body/hydrodynamic simulations that include radiative cooling with the public version of the Hydra code, modified to consider an arbitrary dark-energy component. We produce cluster catalogues for the four models and derive the associated $\mathrm{X}$-ray and SZ scaling relations.

Results. We find that dark energy has little effect on scaling laws, making it safe to use the standard $\Lambda$ CDM scalings for conversion of observed quantities into cluster temperatures and masses.
\end{abstract}

Key words. large-scale structure of Universe

\section{Introduction}

To explain the current acceleration of the universe (Riess et al. 1998; Perlmutter et al. 1999) in the context of the theory of General Relativity, it is general procedure to introduce a new form of gravitational component with negative pressure - dark energy. Various candidates, such as a cosmological constant or a quintessence field, have been proposed. These models are characterised by their equation-of-state parameter and constrained by either using the observation of background quantities or the growth of cosmic structures. An alternative way of explaining the acceleration of the universe is to allow for modifications of gravity. Many classes of models exist; for instance, a light scalar field coupled to matter leads to models of extended quintessence and more generally to scalar-tensor type theories. Other possibilities were studied in the context of braneworld models. Testing the Poisson equation on large scales may be a way of distinguishing between all these alternative scenarios.

Regardless of its nature, dark energy as a dominant component plays a role in structure formation and thus modifies the number of formed structures. The evolution of linear perturbations in the presence of a scalar field, such as quintessence and the effects on the abundance of collapsed structures and its dependence on redshift, were widely explored and suggested as a tool for constraining the nature of dark energy and its evolution (Haiman et al. 2001; Weller et al. 2001; Weinberg \& Kamionkowski 2003; Battye \& Weller 2003; Wang et al. 2004; Mohr 2004). The properties of collapsed haloes (density contrast and virial radius) depend strongly on the shape of the potential, the initial conditions, the time evolution of the dark energy equation of state, and on its ability to collapse on the structure scale (Nunes \& Mota 2006). One can investigate the effects of dark energy on the growth rate of structure and consequently study how dark energy affects the abundance of collapsed halos in dark energy models (Nunes et al. 2006; Manera \& Mota 2006).

Cluster number counts can potentially distinguish between dark energy models, in particular, the evolution of their equation of state. However, a large number of clusters with known redshifts from SZ, X-ray, or optical surveys are needed (Bartelmann et al. 2005). Future Sunyaev-Zel'dovich (SZ), as well as X-rays observations, will provide us with high quality data making galaxy clusters an efficient and powerful cosmological tool. Cluster-physics, however, is complex. The presence of substructures, the possible contamination by radio and IR sources, the still imperfect knowledge of the relation between the halo mass, and the clusters' observed properties induce degeneracies between cluster physics and cosmological models. Scaling relations are key quantities in observational cosmology as they 
relate the observables in X-rays and SZ to the cluster properties, namely, masses and temperatures. The latter are then used in cluster number counts to constrain the cosmological models.

Due to their importance in translating observations to physical quantities, but most of all in probing the cluster formation, scaling relations have raised considerable attention. Simple models of formation of virialised, systems such as clusters predict that they exhibit self-similar behaviours (Kaiser 1986), see also Ascasibar et al. (2006). In the self-similar model, gravitational infall drives shock heating of the intra-cluster medium (ICM) and establishes the gas properties, such that they scale with the halo mass giving rise to the scaling relations. However, it is now clear that more physics is required to provide a more complete picture of the cluster formation and evolution and to explain the deviations between observations and the predictions based on self-similar scaling as shown mostly by X-ray observations (Edge \& Stewart 1991; Allen \& Fabian 1998; Markevitch 1998; Nevalainen et al. 2000; Finoguenov et al. 2001; Ettori et al. 2004; Henry 2004; Arnaud et al. 2005; Rasia et al. 2005; Balogh et al. 2006; Maughan et al. 2006; Morandi et al. 2007). As the complexity of the physical description increases due to the additional gas physics (galactic winds and/or quasar outflows, radiative cooling, preheating), the use of numerical simulations appears the best option for comparing predictions and observations and examining the role of those new ingredients in explaining the departures form self similarity (Evrard et al. 1996; Bryan \& Norman 1998; Bialek et al. 2001; Thomas et al. 2001; Babul et al. 2002; Voit et al. 2002; Borgani et al. 2004; Rowley et al. 2004; Muanwong et al. 2006; Kay et al. 2007). The ICM can also be probed by the SunyaevZel'dovich (SZ) effect, inverse Compton scattering of cosmic microwave background (CMB) photons off high-energy electrons. The magnitude of the SZ effect is determined by the integrated gas pressure along the line-of-sight, called the Compton parameter, $y$. Unlike the X-ray surface brightness, the SZ effect is not subject to cosmological dimming and can be used out to high redshift, which makes SZ-scaling relations particularly interesting and attractive tests. Nevertheless, although gaining in quality and quantity, SZ measurements are at the moment still not sufficient to be fully used and this explains why investigations of SZ scaling relations are still limited from the observational point of view (Cooray 1999; Benson et al. 2004; McCarthy et al. 2003; Morandi et al. 2007), as well as from the numerical point of view (da Silva et al. 2004; Molt et al. 2005; Bonaldi et al. 2007).

Scaling laws relate the observed properties in X-rays and SZ to the cluster properties, namely, masses and temperatures that are then used to construct mass functions and number counts utilised to constrain the cosmological models. Understanding the possible biases in the scaling relations is thus essential for the use of clusters as cosmological probes. Previous studies based on numerical simulations have focused on the effects of additional gas physics in the scaling laws. In the present study, we explore the effects of the cosmological model on the scaling properties of galaxy clusters and their evolutions. We perform hydrodynamic numerical simulation of cluster formation and evolution assuming a simple radiative cooling model, rather than a more complete gas model, which allows us to single out only darkenergy properties. We then investigate whether the X-ray and SZ scaling laws derived for different dark-energy models depart from those obtained in the standard cosmological constant model (the $\Lambda \mathrm{CDM}$ model) taken as our reference. In the next section we briefly present the dark-energy models used for the first numerical simulation of cluster formation with hydrodynamics in dark-energy dominated universes. We describe the simulation code and the procedure used to construct the X-ray and SZ cluster catalogues. In Sect. 3, we present the X-ray and SZ scaling laws studied in the article, together with the fitting procedure. Our results and conclusions are summarised in Sects. 4 and 5, respectively.

\section{Dark-energy simulations}

\subsection{Simulation models}

Numerical $N$-body simulations including a dark-energy component were performed by several groups to complement the analytical computations of structure formation in the presence of dark energy, and to the study the effects of dark energy at the structure level. All studies on galaxy clusters, were essentially dedicated to studying dark-matter halo shapes and mass functions in different models of dark energy (Linder \& Jenkins 2003; Lokas et al. 2003; Klypin et al. 2003; Dolag et al. 2004; Kuhlen et al. 2004). The overall picture that has emerged from these studies is that halo mass functions are well approximated by the Jenkins mass function (Jenkins et al. 2001) and halo core densities, or concentrations, are sensitive to the mean cosmological density at halo formation and therefore depend on the underlying dark-energy model. These findings have led to investigations of the effect of dark energy on the lensing properties of simulated cluster sized haloes and their possible use for constraining dark energy (Meneghetti et al. 2005a,b). In the present study, we perform the first hydrodynamics simulation of cluster formation in dark-energy dominated universes and we focus, for the first time, on the baryonic component of clusters and investigate the possible effects of dark energy on their gas properties. In an earlier study, Maio et al. (2006) produced numerical simulations of dark energy with baryonic gas to study the implications on cosmic reionisation from first stars.

In all the aforementioned studies, the scalar field associated with dark energy is assumed not to have density fluctuations on scales of galaxy clusters or below. If dark energy influences the perturbations on small scales as proposed by Arbey et al. (2001), Bean \& Magueijo (2002), Padmanabhan \& Choudhury (2002), or Bagla et al. (2002), the collapse of structures itself will be affected. In our simulations, we also ignore any possibility of dark energy clustering and influencing the cluster formation.

In addition to the cosmological constant model with a constant equation-of-state parameter $w=\rho_{\mathrm{de}} / P_{\mathrm{de}}=-1$, we simulated four other models previously studied in Nunes et al. (2006). These phenomenological models span the range of values that the equation-of-state parameter can take for typical quintessence models. All models are compatible with current observational constraints at $z \simeq 0$, but they differ especially in the range of redshift of interest for cluster formation $(z<5)$. This is clearly illustrated in Fig. 1 of Nunes et al. (2006). Namely, we take two models for which the dark energy is given by a perfect fluid with constant equation-of-state parameter: $w=-0.8$ and $w=-1.2$ (phantom dark energy), and one model where dark energy results from a slowly evolving scalar field in a potential with two exponential terms (2EXP1, Barreiro et al. 2000)

$V(\phi)=V_{0}\left(\mathrm{e}^{\alpha \kappa \phi}+\mathrm{e}^{\beta \kappa \phi}\right)$

where $\alpha=6.2$ and $\beta=0.1$, which has an equation-of-state parameter today, of $w_{0}=-0.95$. One other model (2EXP2) with a varying equation of state was studied in Nunes et al. (2006). However, because its energy contribution to the total 

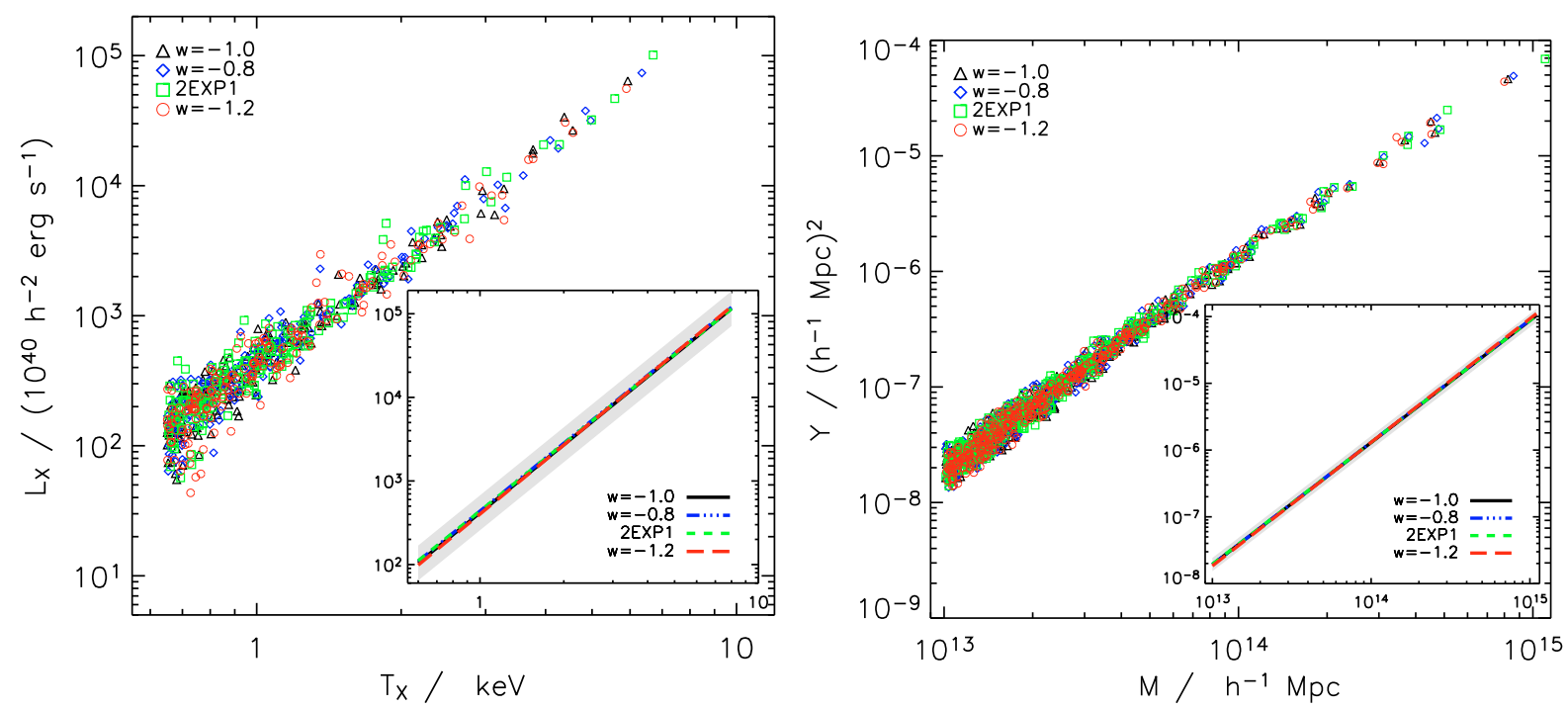

Fig. 1. Cluster scaling relations $L_{\mathrm{X}, 200}-T_{\mathrm{X}, 200}$ (left panel) and $Y_{200}-M_{200}$ (right panel) at redshift zero. Displayed quantities are computed within $R_{200}$, the radius where the mean cluster density is 200 times higher than the critical density. The embedded plots show the best fits with a power law to clusters represented in the main plots for the $w=-1$ (triangles), $w=-0.8$ (diamonds), 2EXP1 (squares), and $w=-1.2$ (circles) models. The shaded regions in the embedded plots give the typical scatter of the fits, i.e. the rms dispersion around the best-fit lines.

energy of the universe quickly decays with redshift, it has no significant departures from the Lambda model in the redshift range of cluster formation (e.g. $z<5$ ). We thus choose, from this point onwards, not to consider this model it in this work. We further assume for all models that the energy density of dark energy, dark matter, and baryons today are, respectively, $\Omega_{\mathrm{de}}=0.7, \Omega_{\mathrm{m}}=0.3, \Omega_{\mathrm{B}}=0.0486$, and the Hubble parameter $h=H_{0} / 100 \mathrm{~km} \mathrm{~s}^{-1} \mathrm{Mpc}^{-1}=0.7 . \sigma_{8}=0.9$.

\subsection{The simulation code}

For each of the cosmological models considered, we performed $\mathrm{N}$-body/hydrodynamic simulations of structure formation using a modified version of the public Hydra code (Couchman et al. 1995; Pearce \& Couchman 1997), which implements an adaptive particle-particle/particle-mesh $\left(\mathrm{AP}^{3} \mathrm{M}\right)$ algorithm to calculate gravitational forces (Couchman 1991) and smoothed particle hydrodynamics (SPH) (Monaghan 1992) to estimate hydrodynamic quantities. The SPH implementation follows that used by Thacker \& Couchman (2000) and conserves both energy and entropy. All simulations analysed below include a model for radiative cooling using the method described in Thomas \& Couchman (1992) and based on the cooling tables of Sutherland \& Dopita (1993). The metallicity was assumed to be a global time varying quantity, $Z=0.3\left(t / t_{0}\right) Z_{\odot}$, where $t / t_{0}$ is the age of the universe in units of the current time and $Z_{\odot}$ is the solar metalicity. At a given time step, gas particles with temperatures below $1.2 \times 10^{4} \mathrm{~K}$ and overdensities (relative to the critical) higher than $10^{4}$ are converted into collisionless material and no longer participate in the gas's dynamical processes.

We modified the computation of the physical time, $t$, and scale factor, $a$, in Hydra to account for the effect of time-variable equations of state of dark energy,

$t=\int_{0}^{a} \frac{\mathrm{d} a^{\prime}}{a^{\prime} H\left(a^{\prime}\right)}=H_{0}^{-1} \int_{0}^{a} \frac{\mathrm{d} \ln a^{\prime}}{E\left(a^{\prime}\right)}$,

where $E(a) \equiv H(a) / H_{0}$, or

$E(a)^{2}=\frac{\Omega_{\mathrm{m}}}{a^{3}}+\Omega_{\mathrm{de}} \mathrm{e}^{-3 \int_{a}^{1}\left(1+w\left(a^{\prime}\right)\right) \frac{\mathrm{d} a^{\prime}}{a^{\prime}}}+\frac{1-\Omega_{\mathrm{m}}-\Omega_{\mathrm{de}}}{a^{2}}$, for flat cosmologies. To speed up computations, this quantity is pre-tabulated for each of the dark-energy models and read once at the beginning of the simulation run. Then $E(a)$ is interpolated and used in Eq. (2). With these modifications, our version of the Hydra code can thus be used to simulate generic models of homogeneous dark energy.

The initial density field of simulations was constructed, at redshift $z=49$, using $N=4096000$ particles of baryonic and dark matter, perturbed from a regular grid of fixed comoving size $L=100 \mathrm{~h}^{-1} \mathrm{Mpc}$. We used the Zel'dovich approximation and the same set of random numbers to generate the initial displacements. The amplitude of the matter power spectrum was calculated assuming $\sigma_{8}=0.9$ in all models and the linear growth factor computed for each model, as presented in Nunes \& Mota (2006). We refer the reader to Nunes et al. (2006) for a discussion of the normalisation. We also assume that the matter power spectrum transfer function is the same for all models and equals that of the cosmological constant model, which is computed using the BBKS formula (Bardeen et al. 1986) and the shape parameter $\Gamma$ given by the formula in Sugiyama (1995). With this choice of parameters, the dark matter and baryon particle masses are $2.1 \times 10^{10} \mathrm{~h}^{-1} M_{\odot}$ and $2.6 \times 10^{9} \mathrm{~h}^{-1} M_{\odot}$ respectively. In physical units the gravitational softening was set fixed to $25 \mathrm{~h}^{-1} \mathrm{kpc}$ below $z=1$ and above this redshift scaled as $50(1+z)^{-1} \mathrm{~h}^{-1} \mathrm{kpc}$.

Individual simulation runs took between 2592 to 2812 time steps to evolve to $z=0$. For each run we stored a total of 50 simulation snapshots (box outputs) for a list of selected redshifts (the same for all runs) ranging from $z=20$ to $z=0$. Thirty of these outputs are inside the interval $0<z<3$, which is typically the range where galaxy clusters form.

\subsection{Catalogue construction}

From simulations, we constructed cluster catalogues using a modified version of the cluster extraction software developed by Thomas and collaborators (Thomas et al. 1998; Pearce et al. 2000; Muanwong et al. 2001). To summarise, the cluster identification process starts with the construction of a minimal-spanning 
tree of dark-matter particles whose density exceeds the mean density of the box by $\Delta_{\mathrm{b}}=178 \times \Omega_{\mathrm{m}}^{-0.55}(z)$ (i.e., the density contrast predicted by the spherical collapse model of a virialised sphere relative to the mean background density in the Lambda cosmology; Eke Navarro \& Frenk 1998). Although $\Delta_{\mathrm{b}}$ may differ for different dark-energy models, this is not important at this step because cluster properties are computed at fixed overdensities as described below. The minimal-spanning tree is then split into clumps of particles using a maximum linking length equal to $0.5 \Delta_{\mathrm{b}}^{-1 / 3}$ times the mean inter-particle separation. Finally we grow a sphere around the densest dark-matter particle in each clump until the enclosed mass verifies

$M_{\Delta}\left(<R_{\Delta}\right)=\frac{4 \pi}{3} R_{\Delta}^{3} \Delta \rho_{\text {crit }}(z)$,

where $\Delta$ is a fixed overdensity contrast, $\rho_{\text {crit }}(z)=$ $\left(3 H_{0}^{2} / 8 \pi G\right) E^{2}(z)$ is the critical density and $E(z)$ is given by Eq. (3). We have constructed master catalogues for all dark-energy simulation containing at least 500 particles of gas and dark matter, i.e. with an equivalent minimum mass of $M_{\text {lim }} \approx 1.18 \times 10^{13} \mathrm{~h}^{-1} M_{\odot}$, at four fixed overdensities, $\Delta=200,500,1000,2500$. Here we will report our findings for cluster scaling relations only at $\Delta=200$, the highest cluster overdensity radius usually considered in the literature. For this catalogue we find $377,393,396,374$ clusters at $z=0$ in the cosmological constant, $w=-0.8,2 \mathrm{EXP} 1$ and $w=-1.2$ simulation runs respectively. Although our choice of $\Delta_{b}$ may limit any exact comparison of numbers, these abundances reproduce the behaviour predicted in Nunes et al. (2006) in their analytical study. We note that the cluster definition used in this article is different from that used in da Silva et al. (2004) and Muanwong et al. (2006). Despite the similar simulation parameters, direct comparison with their findings is only possible for the Lambda model at redshift zero, where cluster definitions are identical.

For each model, the cluster catalogue provides us with estimated structural and observable quantities. We refer the reader to da Silva et al. (2004) for the definitions of the these quantities. More specifically for this study we compute: masses; intrinsic SZ luminosity, $Y=Y_{\mathrm{SZ}} \times D_{\mathrm{A}}^{2}$ where $Y_{\mathrm{SZ}}$ is the integrated SZ signal and $D_{\mathrm{A}}$ is the angular diameter distance; massweighted gas temperature $T_{\mathrm{mw}}$; and bolometric X-ray temperature, $T_{\mathrm{X}}$, and luminosity, $L_{\mathrm{X}}$, excluding a cooling radius of about $50 \mathrm{~h}^{-1} \mathrm{kpc}$ around the cluster centre. We excised the central region to focus on the properties on the cluster scale and to reduce degeneracies between possible effects of dark energy and the effects of cooling. For the SZ emission, the contribution is dominated by the outer parts of clusters. Differences due to the cooling are thus negligible for the SZ scalings.

\section{Analysis of the scaling relations}

In this paper we investigate the following scaling relations between cluster properties: $T_{\mathrm{X}}-M, Y-M, Y-T_{\mathrm{mw}}, L_{\mathrm{X}}-T_{\mathrm{X}}$, and $Y-L_{\mathrm{X}}$. These can be expressed as

$$
\begin{aligned}
& T_{\mathrm{X}}=A_{\mathrm{TM}}\left(M / M_{0}\right)^{\alpha_{\mathrm{TM}}}(1+z)^{\beta_{\mathrm{TM}}} E(z)^{2 / 3}, \\
& Y=A_{\mathrm{YM}}\left(M / M_{0}\right)^{\alpha_{\mathrm{YM}}}(1+z)^{\beta_{\mathrm{YM}}} E(z)^{2 / 3}, \\
& Y=A_{\mathrm{YT}}\left(T_{\mathrm{mw}} / T_{\mathrm{mw}, 0}\right)^{\alpha_{\mathrm{YT}}}(1+z)^{\beta_{\mathrm{YT}}} E(z)^{-1}, \\
& L_{\mathrm{X}}=A_{\mathrm{LT}}\left(T_{\mathrm{X}} / T_{\mathrm{X}, 0}\right)^{\alpha_{\mathrm{LT}}}(1+z)^{\beta_{\mathrm{LT}}} E(z), \\
& Y=A_{\mathrm{YL}}\left(L_{\mathrm{X}} / L_{\mathrm{X}, 0}\right)^{\alpha_{\mathrm{YL}}}(1+z)^{\beta_{\mathrm{YL}}} E(z)^{-9 / 4},
\end{aligned}
$$

where we have chosen the normalisation scales $M_{0}=$ $10^{14} \mathrm{~h}^{-1} M_{\odot}, T_{\mathrm{X}, 0}=T_{\mathrm{mw}, 0}=1 \mathrm{keV}, L_{\mathrm{X}, 0}=10^{43} \mathrm{erg} / \mathrm{s} / \mathrm{h}^{2}$. The powers of the $E(z)$ function give the predicted evolution, extrapolated from the self-similar model (Kaiser 1986), of the scalings in each case. The quantities, $A, \alpha$, and $\beta$, give the scalings normalisation at $z=0$, the power on the independent variable, and the departures from the expected redshift evolution.

To investigate these cluster scaling relations in our simulations, we use the method described in da Silva et al. (2004). According to Eqs. (5)-(9), the general form of how a given cluster property $y$ relates to a property $x$ can be written as

y $f(z)=y_{0}(z)\left(x / x_{0}\right)^{\alpha}$,

where

$y_{0}(z)=A(1+z)^{\beta}$,

and $f(z)$ is some fixed power of the cosmological factor $E(z)$. This is a power-law function whose parameters $A, \alpha$ and $\beta$ can be obtained by fitting our cluster catalogue distributions at each redshift with a straight line in the $(\log (y f(z)), \log x)$ plane. To be more specific, the fitting procedure is carried out in three steps. First, we fit the cluster distributions with a straight line in logarithmic scale at all redshifts. If the logarithmic slope $\alpha$ remains approximately constant (i.e. shows no systematic variations) within the redshift range of interest, we then take $\alpha$ at $z=0$ as the best-fit value. In the second step, we repeat the fit using this value of $\alpha$ to determine the scaling normalisation factors $y_{0}(z)$. This avoids unwanted correlations between $\alpha$ and $y_{0}(z)$. Finally, in the last step we use Eq. (11) to obtain the parameters $A$ and $\beta$.

In the fitting process we consider only clusters with $M_{\lim }>$ $5 \times 10^{13} \mathrm{~h}^{-1} M_{\odot}$ for scalings with mass, $L_{\mathrm{lim}}>6.6 \times$ $10^{42} \mathrm{~h}^{-2} \mathrm{erg} \mathrm{s}^{-1}$ for scalings with luminosity, and $T_{\mathrm{mw}, \lim }>$ $1 \mathrm{keV}, T_{\mathrm{X}, \mathrm{lim}}>1.1 \mathrm{keV}$ for scalings with mass-weighted and emission-weighted temperatures, respectively. This ensures that our cluster samples are complete at all redshifts and that, for each model, equal number of clusters are used for all scalings at redshift zero. With these selection criteria, our cluster samples contain 60 clusters for the cosmological constant model and a similar number of clusters for the other models, at $z=0$. We note that above $z=1.5$ the number of clusters with $M_{\lim }>5 \times 10^{13} \mathrm{~h}^{-1} M_{\odot}$ in our sample decreases typically below 10 , hence; we do not fit the scaling relations above this redshift value. As we discuss in the next section, all the scaling relations explored in the present study are well-fitted by power laws of the form Eq. (10), in the redshift range $0<z<1.5$, except for the $L_{X}-T_{X}$ and $Y-L_{X}$ whose normalisation dependences on redshift, $\log \left(y_{0}(z)\right)$, are approximated well by a straight line only in a narrower redshift range.

\section{Results}

\subsection{Scaling relations at $z=0$}

We start by discussing the cluster scaling laws at redshift zero. For all scalings and models under investigation in this work, these were determined at an overdensity radius $R_{200}$. In Table 1 we present the power-law best fit for the logarithmic slopes $\alpha$ obtained for each of the cases. As can be inferred from the table, all models provide very similar results for each scaling, with differences between models comparable to or, in most cases, within the statistical errors of the fit. This indicates that the local cluster scaling relations are quite insensitive to the underlying darkenergy model driving the present-day evolution of the universe. 
Table 1. Best fit values of the parameters $\alpha, \log A$ and $\beta$ as well as their respective $1 \sigma$ errors.

\begin{tabular}{llrrrr}
\hline \hline Model & & $w=-1$ & $w=-0.8$ & 2EXP1 & $w=-1.2$ \\
\hline$T_{\mathrm{X}}-M$ & $\alpha_{\mathrm{TM}}$ & $0.620 \pm 0.029$ & $0.604 \pm 0.031$ & $0.581 \pm 0.033$ & $0.602 \pm 0.029$ \\
& $\log A_{\mathrm{TM}}$ & $0.260 \pm 0.005$ & $0.267 \pm 0.004$ & $0.271 \pm 0.005$ & $0.258 \pm 0.005$ \\
& $\beta_{\mathrm{TM}}$ & $-0.228 \pm 0.020$ & $-0.249 \pm 0.017$ & $-0.264 \pm 0.023$ & $-0.230 \pm 0.023$ \\
$Y-M$ & $\alpha_{\mathrm{YM}}$ & $1.732 \pm 0.025$ & $1.730 \pm 0.025$ & $1.721 \pm 0.022$ & $1.752 \pm 0.024$ \\
& $\log A_{\mathrm{YM}}$ & $-5.910 \pm 0.004$ & $-5.906 \pm 0.004$ & $-5.902 \pm 0.005$ & $-5.910 \pm 0.003$ \\
& $\beta_{\mathrm{YM}}$ & $0.128 \pm 0.016$ & $0.116 \pm 0.016$ & $0.108 \pm 0.020$ & $0.135 \pm 0.013$ \\
$Y-T_{\mathrm{mw}}$ & $\alpha_{\mathrm{YT}}$ & $2.922 \pm 0.100$ & $2.902 \pm 0.136$ & $2.838 \pm 0.072$ & $2.985 \pm 0.175$ \\
& $\log A_{\mathrm{YT}}$ & $-6.522 \pm 0.008$ & $-6.518 \pm 0.005$ & $-6.499 \pm 0.007$ & $-6.538 \pm 0.008$ \\
& $\beta_{\mathrm{YT}}$ & $0.454 \pm 0.036$ & $0.443 \pm 0.022$ & $0.430 \pm 0.031$ & $0.517 \pm 0.036$ \\
$L_{\mathrm{X}}-T_{\mathrm{X}}$ & $\alpha_{\mathrm{LT}}$ & $2.738 \pm 0.086$ & $2.691 \pm 0.089$ & $2.902 \pm 0.099$ & $2.796 \pm 0.146$ \\
& $\log A_{\mathrm{LT}}$ & $2.602 \pm 0.010$ & $2.629 \pm 0.007$ & $2.558 \pm 0.006$ & $2.589 \pm 0.011$ \\
& $\beta_{\mathrm{LT}}$ & $0.279 \pm 0.063$ & $0.027 \pm 0.042$ & $0.270 \pm 0.035$ & $0.348 \pm 0.070$ \\
$Y-L_{\mathrm{X}}$ & $\alpha_{\mathrm{YL}}$ & $1.063 \pm 0.028$ & $1.064 \pm 0.026$ & $1.076 \pm 0.026$ & $1.084 \pm 0.037$ \\
& $\log A_{\mathrm{YL}}$ & $-6.314 \pm 0.005$ & $-6.330 \pm 0.004$ & $-6.344 \pm 0.005$ & $-6.305 \pm 0.006$ \\
& $\beta_{\mathrm{YL}}$ & $0.668 \pm 0.033$ & $0.890 \pm 0.028$ & $0.770 \pm 0.034$ & $0.497 \pm 0.037$ \\
\hline
\end{tabular}

See text for the redshift ranges in which the linear fit is a good approximation.

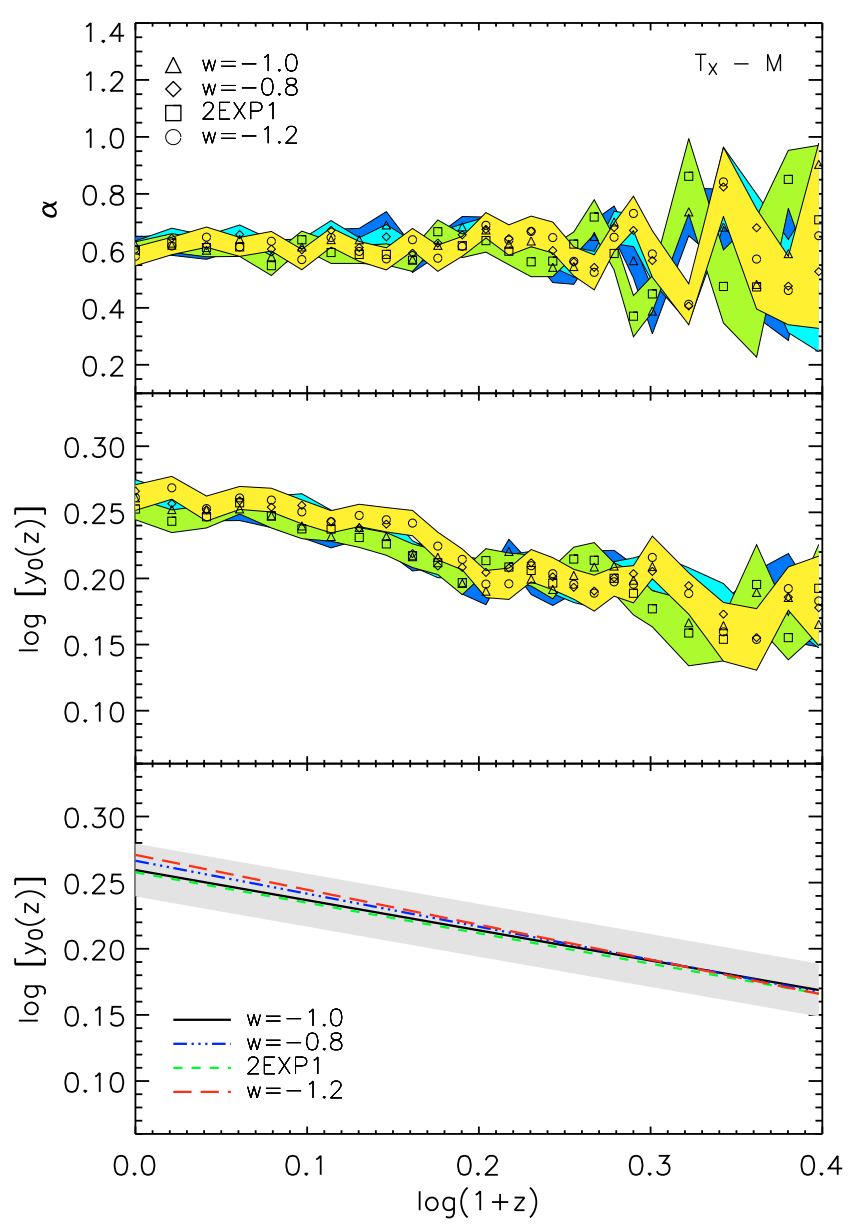

Fig. 2. Slope (upper panel), normalisation $\log \left(y_{0}(z)\right)$ (middle panel), and normalisation best-fit lines (bottom panel) of the $T_{\mathrm{X}}-M$ relation as a function of redshift $\left(\log \left(y_{0}(z)\right)\right.$ is defined in Eq. (11)). Blue colour and triangles stand for the cosmological constant model, cyan, and diamonds are for the $w=-0.8$ model, green and squares are for the 2EXP1 model, and yellow and circles are for the $w=-1.2$ model. The shaded area in the bottom panel gives the dispersion of the normalisation fit for the cosmological-constant model.

As stated in Sect. 2.3, our cluster definition is the same as in da Silva et al. (2004) and Muanwong et al. (2006) at redshift zero for the Lambda model. We verified that indeed theirs and our results are in excellent agreement at this redshift.

To illustrate the robustness of the scalings with respect to the dark-energy models investigated in this paper, we present in Fig. 1 two characteristic X-ray and SZ galaxy cluster scaling relations: the $L_{\mathrm{X}}-T_{\mathrm{X}}$ (left panel) and the $Y-M$ (right panel) scalings. In each case, the main plot shows the cluster distributions for all models, whereas the embedded panels show the powerlaw best fits obtained. For both scaling relations, the cluster distributions and best-fit lines clearly overlap. Also represented by a shaded area in the embedded panels is the rms dispersion of the fit for Lambda model:

$\sigma_{\log y^{\prime}}=\sqrt{\frac{1}{N} \sum_{i}\left(\log \left(y_{i}^{\prime} / y^{\prime}\right)\right)^{2}}$,

where $y^{\prime}=y f$ (see Eq. (10)) and $y_{i}^{\prime}$ are individual data points. This dispersion is the same size as the fit dispersions obtained in the other models and it is clearly wider than the best-fit line separations of the various dark energy models. As expected, the scatter in the (core excised) $L_{\mathrm{X}}-T_{\mathrm{X}}$ is greater than in the $Y-M$ relation due to the higher sensitivity of the former scaling to the gas physics in the inner regions of clusters.

\subsection{Evolution of the scaling relations}

In Figs. 2-6, we present our findings for the variation with redshift of $\alpha$ (top panels) and $y_{0}(z)$ (middle panels) for the $T_{\mathrm{X}}-M$, $Y-M, Y-T_{\mathrm{mw}}, L_{\mathrm{X}}-T_{\mathrm{X}}$, and $Y-L_{\mathrm{X}}$ relations, respectively. The coloured bands give the the best fit errors obtained at each redshift for these quantities. The lines in the bottom panels of these figures are linear best fits to the evolution of $\log \left(y_{0}(z)\right)$ with $\log (1+z)$. The shaded area in these panels gives the rms dispersion of the $\log \left(y_{0}(z)\right)$ fit for the cosmological constant model (similar values of dispersion are found for the other models). The resulting best-fit parameters for, $A, \beta$, and $\alpha$ are presented in Table 1 , for all scaling investigated in this paper.

As can be inferred from the figures, the fit to a power law in a redshift range $0<z<1.5$ was possible for all the scaling relations explored in the present study except the relations involving $L_{\mathrm{X}}$ (namely $L_{\mathrm{X}}-T_{\mathrm{X}}$ and $Y-L_{\mathrm{X}}$ ). For the latter two, we found no significant departures of the slopes $\alpha$ from the cosmological constant model, but we could not fit the evolution of the 


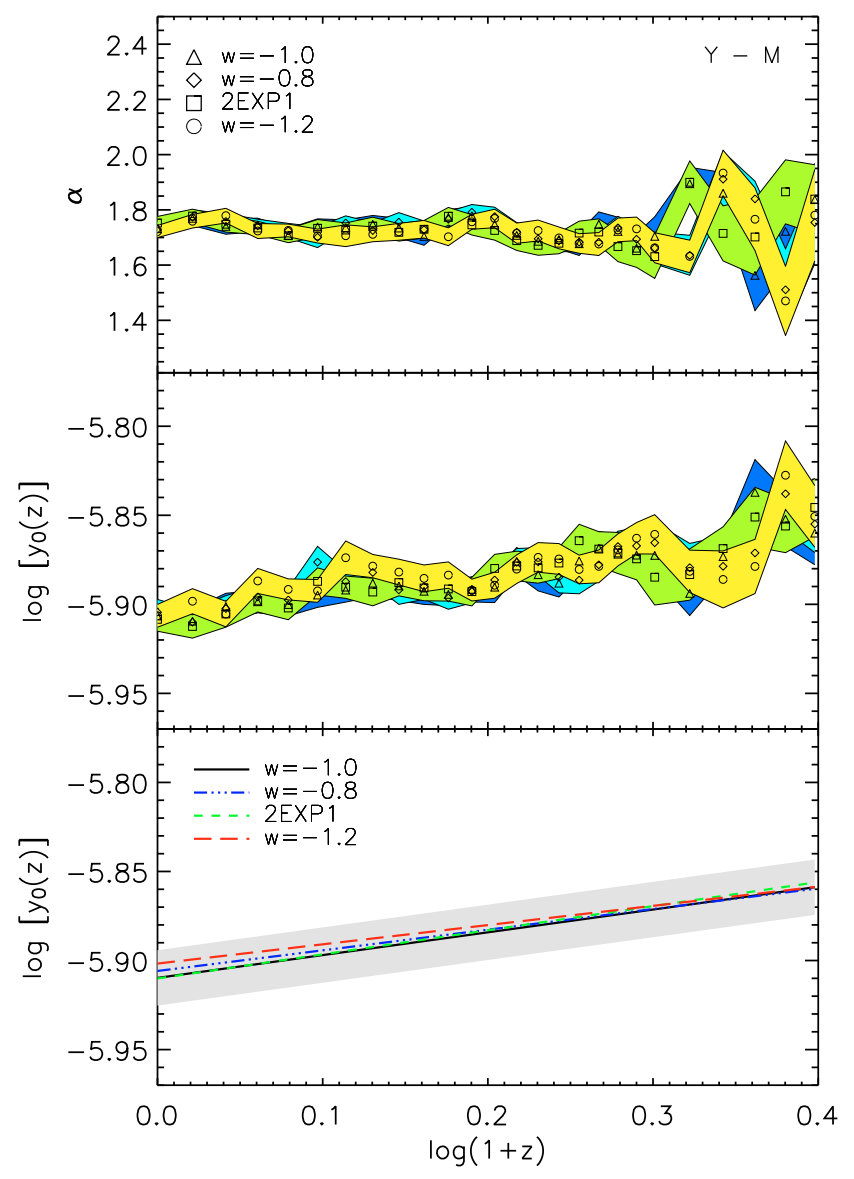

Fig. 3. Slope, normalisation, and normalisation best-fit lines of the $Y-$ $M$ relation as a function of redshift. The shaded area in the bottom panel stands for the dispersion of the normalisation for the Lambda model.

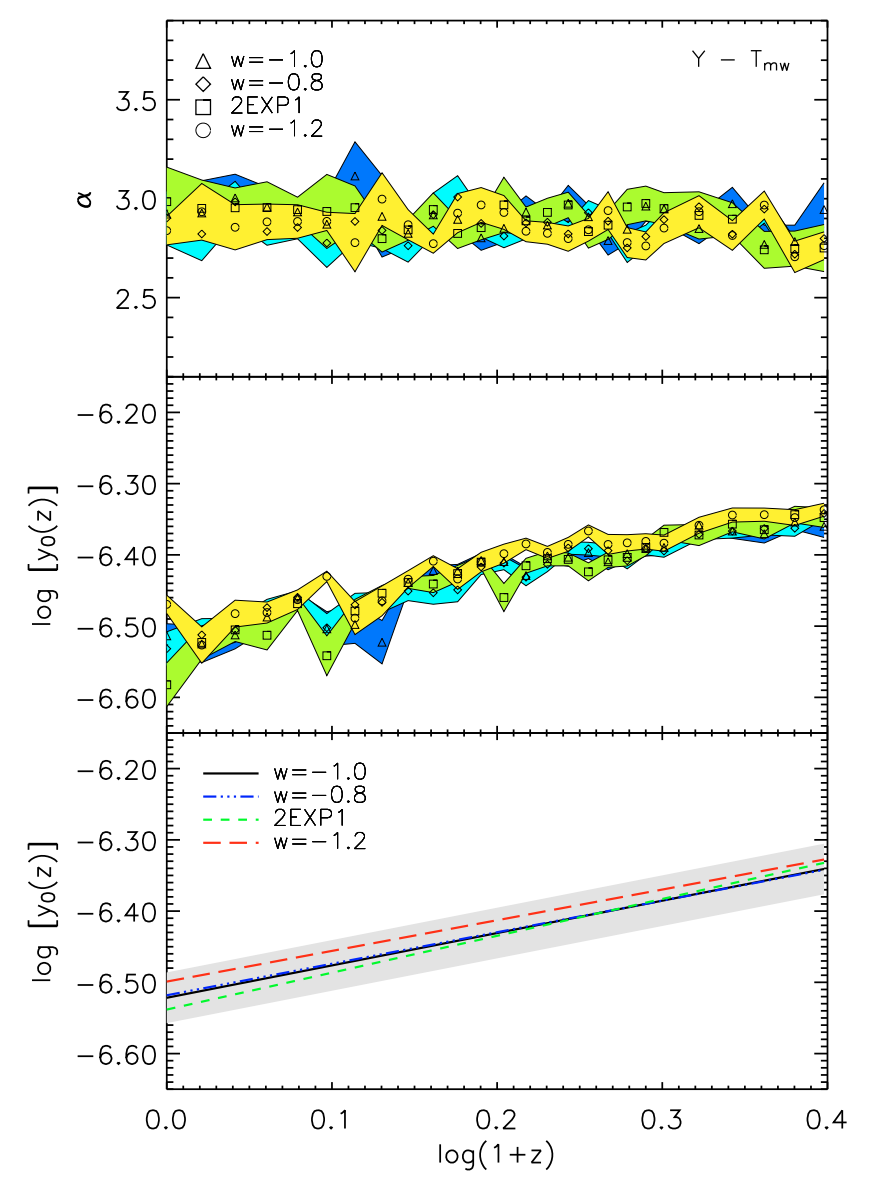

Fig. 4. Slope, normalisation, and normalisation best-fit lines of the $Y-T_{\mathrm{mw}}$ relation as a function of redshift. The shaded area in the bottom panel stands for the dispersion of the normalisation for the Lambda model.

evolution, (da Silva et al. 2004; Muanwong et al. 2006) for studies in cosmological constant model simulations.

It is clear from the table and the lower panels of the figures that the value of $\beta$ is slightly more model dependent than the parameters $\alpha$ and $A$. This is especially true for the $L_{\mathrm{X}}-T_{\mathrm{X}}$, and $Y-L_{\mathrm{X}}$ relations for which there is a mild difference between models. For these scalings the $w=-1.2$ and $w=-0.8$ model generally show the strongest deviations from the cosmologicalconstant model, whereas the 2EXP1 show the least. However, those differences are of the same order of magnitude as the intrinsic errors and dispersions. We can thus safely consider that there are no significant departures from the cosmological constant model.

\section{Conclusions}

The abundance of clusters, their redshift distribution, as well as their clustering, are governed by the geometry of the universe and the power spectrum of the initial density perturbations. Gas physics related to cluster structure and evolution also enters through mapping of the cluster observable (SZ flux or $\mathrm{X}$-ray luminosity) relative to the total mass of the cluster. As a result, galaxy cluster counts can be used as probes of cosmological and cluster properties. However, there are several requirements needed to achieve precise cosmological constraints: (i) advances in understanding the formation and evolution of els of radiative cooling (which is a non-gravitational physical process) causes cluster scaling laws to deviate from self-similar 


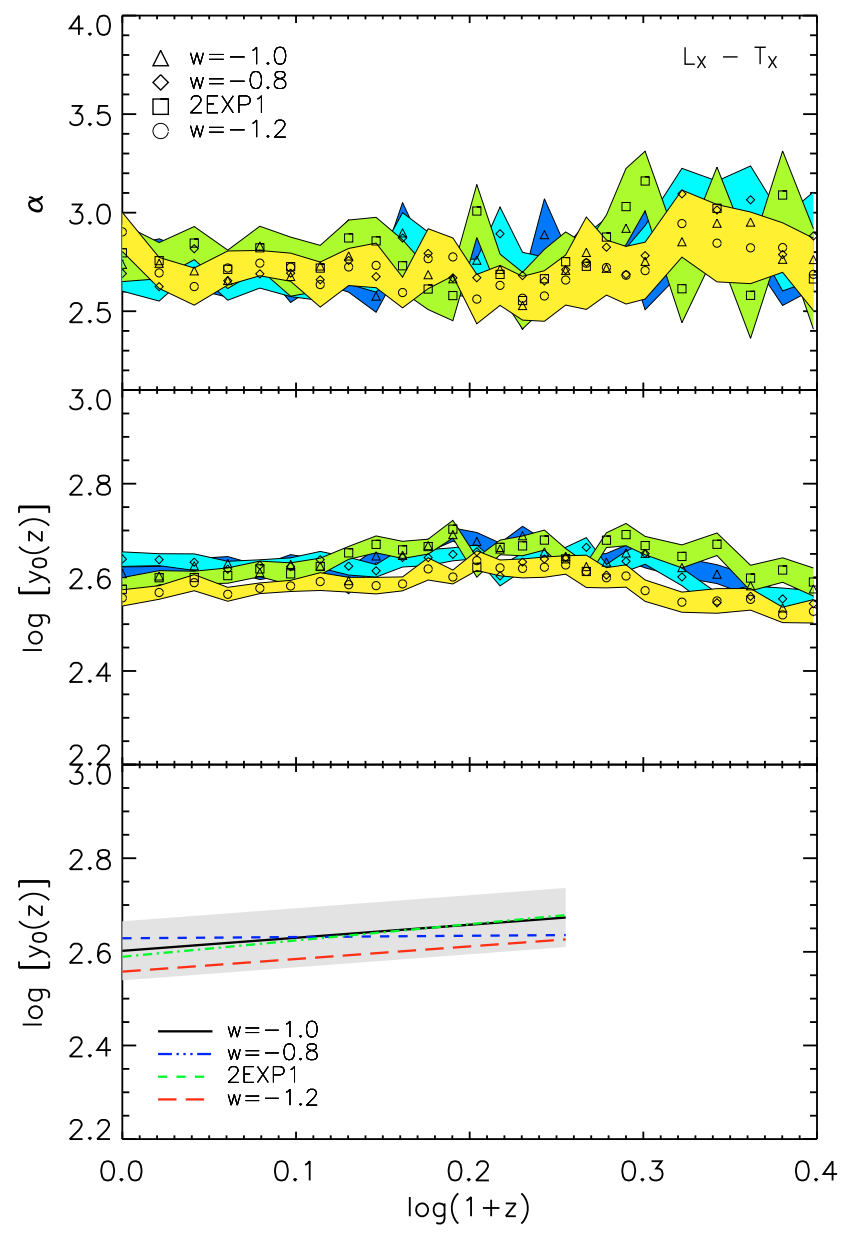

Fig. 5. Slope, normalisation, and normalisation best-fit lines of the $L_{X}-T_{X}$ relation as a function of redshift. The shaded area in the bottom panel stands for the dispersion of the normalisation for the Lambda model.

cluster-size haloes; (ii) a good understanding of the selection function; (iii) robust observational proxies for the cluster mass.

The first condition relates to the conduction of large simulations. This is achieved in the standard cosmological model with a cosmological constant. In the context of a dark-energy dominated universe, $N$-body simulations are becoming available for models different from the simple cosmological constant with constant or varying equation-of-state parameter. These simulations now provide us with a good understanding of the halo properties. They show that the halo mass function is approximated well by the Jenkins mass function. Simulations also indicate that the clusters halos are more concentrated in dark-energy models, since structures grow earlier, than in lambda models and that concentrations are higher in models with varying rather than constant equation state. The second condition, understanding the cluster selection function, translates into understanding the limiting mass and the completeness of the surveys from realistic mock cluster catalogues. The mass (or temperature) selection function is directly linked with the cluster observed quantities through cluster scaling relations, which is our third requirement (the need for a good proxy for the cluster mass).

In this work we have thus for the first time explored the scaling laws for both SZ and X-rays observations using hydrodynamic simulations of galaxy clusters in four dark-energy models with constant or varying equation of states spanning a large

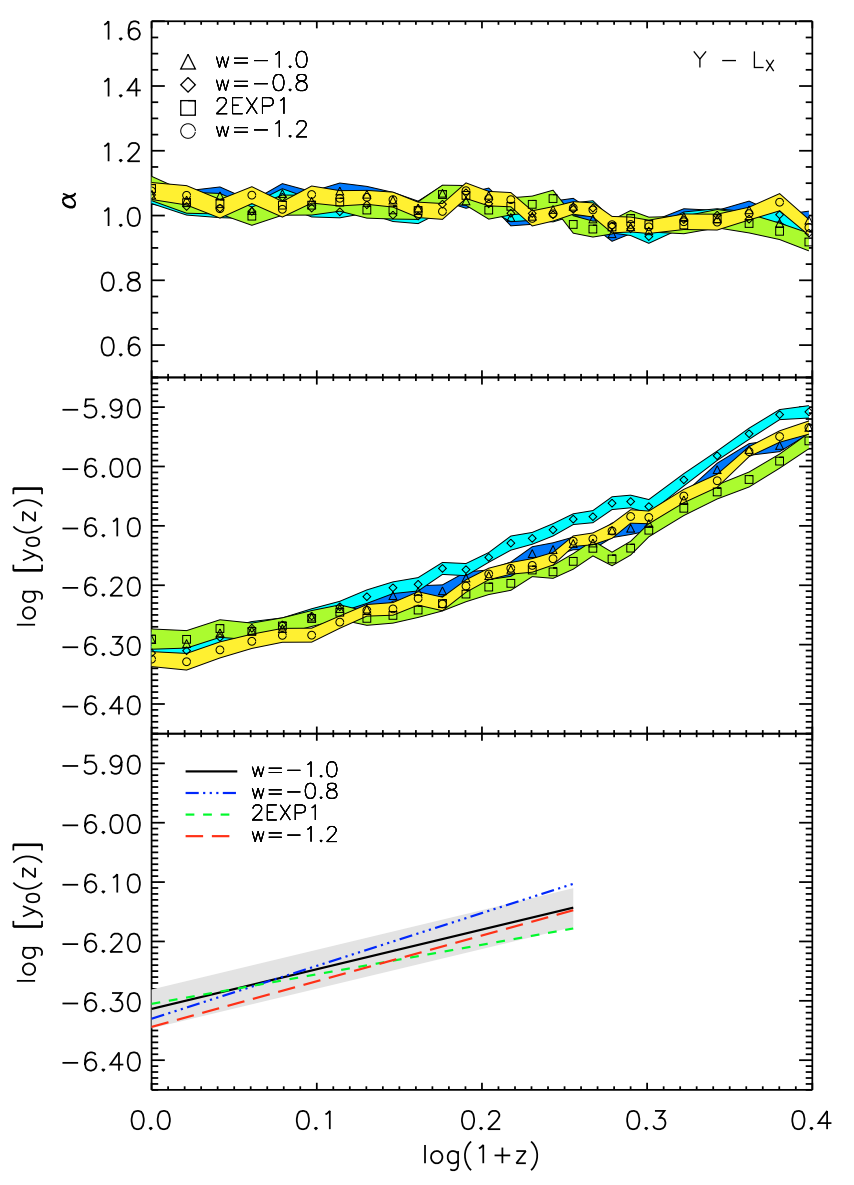

Fig. 6. Slope, normalisation, and normalisation best fit lines of the $Y-L_{\mathrm{X}}$ relation as a function of redshift. The shaded area in the bottom panel stands for the dispersion of the normalisation for the Lambda model.

class of models. We studied the scaling properties at $z=0$ and their evolution with redshift. We find that dark energy induces no modifications on the scaling laws at $z=0$ and presents very little difference from the cosmological-constant model at higher redshifts. The results reported in the article are from simulations with radiative cooling. We also performed adiabatic simulations for the same set of dark-energy models and initial conditions. No significant departures were found in the scalings for the adiabatic runs, thus showing the quasi-null effect of dark energy independently of the cluster physical model.

While detailed simulations incorporating viable dark-energy models remains a programme in progress, it is reassuring that all models considered in this work predict similar scaling properties to the lambda model. The modelling of the cluster gas component appears to be nearly independent of the dark-energy model. Therefore, using the "standard" lambda-model scaling relations for converting observable to masses and temperatures in future surveys should not introduce any additional bias into the cosmological constraints derived from cluster counts. In this work, we have considered that dark energy does not cluster with dark matter. It would be interesting, however, to evaluate how our conclusions stand for numerical simulations in a scenario where dark energy is inhomogeneous and collapses along with dark matter during the formation of structure. This will be pursued in a forthcoming study.

Acknowledgements. The authors are indebted to Peter Thomas, Orrarujee Muanwong, and collaborators for the their part in writing the original Sussex cluster extraction software used in this work. We thank Orrarujee Muanwong 
for discussions and comments on the manuscript. We also acknowledge the PAI-PESSOA collaboration programme as well as a partial support from the CNES and Programme National de Cosmologie. The simulations used in this study were performed at the IAS computing facilities. NA thanks the CAUP for hospitality. N.J.N. is supported by the STFC. AdS acknowledges support from Fundação Ciência e Tecnologia (FCT) under the contracts SFRH/BPD/20583/2004 and CIÊNCIA 2007.

\section{References}

Allen, S. W., \& Fabian, A. C. 1998, MNRAS, 297, L57

Arbey, A., Lesgourgues, J., \& Salati, P. 2001, Phys. Rev. D, 64, 123528

Arnaud, M., Pointecouteau, E., \& Pratt, G. W. 2005, A\&A, 441, 893

Ascasibar, Y., Sevilla, R., Yepes, G., Mueller, V., \& Gottloeber, S. 2006, MNRAS, 371, 193

Babul, A., Balogh, M. L., Lewis, G. F., \& Poole, G. B. 2002, MNRAS, 330, 329

Bagla, J. S., Jassal, H. K., \& Padmanabhan, 2003, Phys. Rev. D, 67, 063504

Balogh, M. L., Babul, A., Voit, M., et al. 2006, MNRAS, 366, 624

Bardeen, J. M., Bond, J. R., Kaiser, N., \& Szalay, A. S. 1986, ApJ, 304, 15

Barreiro, T., Copeland, E. J., \& Nunes, N. J. 2000, Phys. Rev. D, 61, 127301

Bartelmann, M., Doran, M., \& Wetterich, C. 2005

[arXiv: astro-ph/0507257]

Battye, R. A., \& Weller, J. 2003, Phys. Rev. D, 68, 083506

Bean, R., \& Magueijo, J. 2002, Phys. Rev. D, 66, 063505

Benson, B. A., Ade, P. A. R., \& Bock, J. J., et al. 2004, ApJ, 617, 829

Bialek, J. J., Evrard, A. E., \& Mohr, J. J. 2001, ApJ, 555, 597

Bonaldi, A., Tormen, G., Dolag, K., \& Moscardini, L. 2007 [arXiv:0704.2535]

Borgani, S., Murante, G., Springel, V., et al. 2004, MNRAS, 348, 1078

Bryan, G. L., \& Norman, M. L. 1998, ApJ, 495, 80

Cooray, A. R. 1999, MNRAS, 307, 841

Couchman, H. M. P. 1991, ApJ, 368, L23

Couchman H. M. P., Thomas, P. A., \& Pearce, F. R., 1995, MNRAS, 452, 797

Dolag, K., Bartelmann, M., Perrotta, F., et al. 2004, MNRAS, 416, 853

Edge, A. C., \& Stewart, G. C. 1991, MNRAS, 252, 414

Eke, V. R., Navarro, J. F., \& Frenk, C. S. 1998, ApJ, 503, 569

Ettori, S., Tozzi, P., Borgani, S., \& Rosatti, P. 2004, A\&A, 417, 13

Evrard, A. E., Metzler, C. A., \& Navarro, J. F. 1996, ApJ, 469, 494

Finoguenov, A., Reiprich, T. H., Bohringer, H. 2001, A\&A, 368, 749

Haiman, Z., Mohr, J. J., \& Holder, G. P. 2001, ApJ, 553, 545

Henry, J. P. 2004, ApJ, 609, 603

Jenkins, A., Frenk, C. S., White, S. D. M., et al. 2001, MNRAS, 321, 372

Kaiser, N. 1986, MNRAS, 222, 323

Kay, S. T., da Silva, A. C., Aghanim, N., et al. 2007, MNRAS

Klypin, A., Maccio, A. V., Mainini, R., \& Bonometto, S. A. 2003, ApJ, 599, 31

Kuhlen, M., Strigari, L. E., Zentner, A. R., Bullock, J. S., \& Primack, J. R. 2004 [arXiv: astro-ph/0402210]

Linder, E. V., \& Jenkins, J. 2003 [arXiv: astro-ph/0305286]
Lokas, E. L., Bode, P., \& Hoffman, Y. 2003 [arXiv: astro-ph/0309485]

Maio, U., Dolag, K., Meneghetti, M., et al. 2006, MNRAS, 373, 869

Manera, M., \& Mota, D. F. 2006 MNRAS, 371, 1373

Markevitch, M. 1998, ApJ, 504, 27

Maughan, B. J., Jones, L. R., Ebeling, H., \& Scharf, C. 2006, MNRAS, 365, 509

McCarthy, I. G., Babul, A., Holder, G. P., \& Balogh, M. L. 2003, ApJ, 591, 515

Meneghetti, M., Jain, B., Bartelmann, M., \& Dolag, K. 2005a, MNRAS, 362, 1301

Meneghetti, M., Bartelmann, M., Dolag, K., et al. 2005b, A\&A, 442, 413

Mohr, J. J. 2004 [arXiv:astro-ph/0408484]

Molt, P. M., Hallman, E. J., Burns, J. O., \& Norman, M. L. 2005, ApJ, 623, L63

Monaghan, J. J. 1992, ARA\&A, 30, 543

Morandi, A., Ettori, S., \& Moscardini, L. 2007 [arXiv: 0704 . 2678]

Muanwong, O., Thomas, P. A., Kay, S. T., Pearce, F. R., \& Couchman, H. M. P. 2001, ApJ, 552, L27

Muanwong, O., Thomas, P. A., Kay, S. T., \& Pearce F. R., 2002, MNRAS, 336, 527

Muanwong, O., Kay, S. T., \& Thomas, P. A. 2006, ApJ, 649, 640

Nevalainen, J., Markevitch, M., \& Forman, W. 2000, ApJ, 532, 694

Nunes, N. J., \& Mota, D. F. 2006, MNRAS, 368, 751

Nunes, N. J., da Silva, A. C., \& Aghanim, N. 2006, A\&A, 450, 899

Padmanabhan, T., \& Choudhury, T. R. 2002, Phys. Rev. D, 66, 081301

Pearce, F. R., \& Couchman, H. M. P. 1997, New Astron., 2, 411

Pearce, F. R., Thomas, P. A., Couchman, H. M. P., \& Edge, A. C. 2000, MNRAS, 317,1029

Perlmutter, S., Aldering, G., Goldhaber, G., et al. 1999, ApJ, 517, 565

Rasia, E., Mazzotta, P., Borgani, S., et al. 2005, ApJ, 618, L1

Riess, A. G., Filippenko, A. V., \& Challis, P. 1998, AJ, 116, 1009

Rowley, D. R., Thomas, P. A., \& Kay, S. T. 2004, MNRAS, 352, 508

da Silva, A. C., Barbosa, D., Liddle, A. R., \& Thomas, P. A. 2000, MNRAS, 317,37

da Silva, A. C., Barbosa, D., Liddle, A. R., \& Thomas, P. A. 2001, MNRAS, 326, 155

da Silva, A. C., Kay, S. T., Liddle, A. R., \& Thomas, P. A. 2004, MNRAS, 348, 1401

Sugiyama, N. 1995, ApJS, 100, 281

Sunyaev, R. A., \& Zel'dovich, Ya. B. 1972, Comm. Astrophys. Space Phys., 4, 173

Sunyaev, R. A., \& Zel'dovich, Ya. B. 1980, ARA\&A, 18, 537

Sutherland, R. S., \& Dopita, M. A. 1993, ApJS, 88, 253

Thacker, R. J., \& Couchman, H. M. P. 2000, ApJ, 545, 728

Thomas, P. A., \& Couchman, H. M. P. 1992, MNRAS, 257, 11

Thomas, P. A., Colberg, J. M., \& Couchman, H. M. P. (the Virgo Consortium) 1998, MNRAS, 296, 1061

Thomas, P. A., Muanwong, O., Pearce, F. R., et al. 2001, MNRAS, 324, 450

Voit, G. M., Bryan, G. L., Balogh, M. L., \& Bower, R. G. 2002, ApJ, 576, 601

Wang, S., Khoury, J., Haiman, Z., \& May, M. 2004, Phys. Rev. D, 70, 123008

Weinberg, N. N., \& Kamionkowski, M. 2003, MNRAS, 341, 251

Weller, J., Battye, R., \& Kneissl, R. 2002, Phys. Rev. Lett., 88, 231301 